Available online at GSC Online Press Directory

GSC Biological and Pharmaceutical Sciences

e-ISSN: 2581-3250, CODEN (USA): GBPSC2

Journal homepage: https://www.gsconlinepress.com/journals/gscbps

(SHORT COMMUNICATION)

\title{
Think before use: Sterility checking of culture plates
}

\author{
Prabhusaran Nagarajan ${ }^{1 *}$, Pramila Mahalingam ${ }^{2}$ and Anupriya Aasaithambi ${ }^{1}$ \\ ${ }^{1}$ Department of Microbiology, Trichy SRM Medical College Hospital and Research Centre, Tiruchirapalli, India (Affiliated \\ to The Tamilnadu Dr. M.G.R. Medical University, Chennai). \\ 2 Department of Biotechnology, Nehru Memorial College, Tiruchirapalli, India.
}

Publication history: Received on 28 August 2018; revised on 14 September 2018; accepted on 21 September 2018

Article DOI: https://doi.org/10.30574/gscbps.2018.5.1.0094

\begin{abstract}
Microbial contaminations in the culture plates are quite common in microbiological laboratory. The preparation of culture plates is an art thereby the scientific principles should be adhered strictly. While preparing the culture plates, chance of contamination is high either due to inadequate sterilization or improper handling. In general, the prepared plates should not use or store at low temperature immediately. The sterility checking is very important before impregnation the plates for microbiological procedures. This study is aimed to analyze the discarding criteria of the contaminated plates either partly or completely. Two sets of nutrient agar plates were prepared and kept for sterility checking overnight at room temperature. The type of contaminants were assessed; thereby colonies on the surface or underneath the surface. Both the sets were kept for another day for the development of contamination in the contaminant free plates recorded on the day 1 . The results revealed that two types of contaminants were found in two sets. Set one have the colonies on the surface and no further contamination found on day 2 in the non-contaminated plates indicated that the contamination may occur during plating procedures. Set two have the colonies underneath the surface and contamination developed on day 2 in the non-contaminated plates due to improper sterilization of the glassware and culture media. Thus from this study, it was recommended that the set one plates which are not contaminated are used for further processing, whereas the entire set two should be discarded. The major significance of this work is to understand the importance of sterility checking before using the nutrient plates for culturing.
\end{abstract}

Keywords: Culture plates; Sterility; Colonies; Surface; Underneath

\section{Introduction}

Microorganisms are ubiquitous in nature and are considered as free living, saprophytic and parasitic. The existence, growth and development of the microorganism are mostly depending on nutrients and growth enhancing environment [1-2]. As a whole, the nutrients that are promoting the microbial growth are defined as culture media. For isolation and identification of the unknown microbes or to assess their characteristic features, the solid culture media impregnated with agar is recommended. This agar plates afford large surface area for the study of discrete colonies in order to isolate and identify the microbes. The environmental factors must also be regulated including $\mathrm{pH}$, temperature, osmotic pressure, moisture, aerobic and anaerobic conditions [3-4].

Additionally, sterility is the trait of the successful preparation of the culture media. To achieve it, the glasswares and culture media should be sterilized appropriately by following dry and moist heat sterilization respectively [1, 5]. In previous days, the culture media are dispensed in the petridishes in the surface sterilized table top. Even now in the industries while preparing bulk preparations of the culture media plates, the bench top is used. On comparing with other methods, plating provides discrete colonies (individual and macroscopically visible mass of microbial growth and pure culture) that are suitable for studying cultural, morphological and biochemical properties [6-7].

\footnotetext{
${ }^{*}$ Corresponding author

E-mail address: leptoprabhu@gmail.com
} 
Sterilization and aseptic handling are the backbone of the culture media preparation thereby contamination rates get reduced. Contamination is the major issue that is largely affecting the whole process economically and time [2, 8]. Microorganisms that are present in the air, laboratory surfaces including working benches, equipments are largely involved in the contamination of culture media. Thus, proper sterilization of glasswares, chemicals, surfaces and supportive instruments (pipettes, inoculation loops, glass rods etc) are very important to overcome such contaminations $[4,9]$.

For the successful culmination of microbial growth, the culture media plates should not be used immediately after dispersion of the media in petridish. The prepared plates should be kept at room temperature or at incubator for 18 to 24 hours for sterility checking [10-11]. If the plates are used immediately, we cannot predict that the preparation is $100 \%$ sterile, thus chance of getting contamination that disturbs our objectives. Thus selection and discarding the contaminated plates is very important. The inclusion criteria of discarding contaminated plates are improper plating, improper solidification and contamination.

Blindly, in most of the laboratory only the contaminated plates are discarded and uncontaminated plates are used further. In this situation, the following concepts may be adopted. i) Discarding the entire set of contaminated and uncontaminated plates, ii) Discarding the partial number plates that are contaminated and iii) Utilization and/or discarding uncontaminated plates.

The first and second concepts are direct and largely followed in laboratories. The new concept of selection for utilizing or discarding the uncontaminated plates is now emerged and time of need. For exploring that concept, this study has aimed to analyze the discarding criteria of the contaminated plates either partly or completely.

\section{Material and methods}

\subsection{Study type}

This is an observational study where the contamination types were recorded while preparing the bulk culture plates for research purposes.

\subsection{Preparation of culture plates}

The research scholar was indulged to prepare nutrient agar plates in bulk. Proper sterilization procedures including appropriate inclusion of moist and dry heat were followed. After sterilization, the plates were prepared in the laminar air flow by surface sterilizing using chemical and UV light before processing. Air flow was put on while transferring the nutrient media onto the petridishes.

\subsection{Sterility checking}

In general, all the prepared plates were kept at room temperature or incubated for 18 to 24 hours for sterility checking. This procedure was followed by the research scholar for this occasion also.

\subsection{Observation of colonies}

After appropriate time and before using the prepared plates for culturing, contamination status of the plates was recorded. The region, type and flow of contamination was documented as follows, i) No contamination, ii) Contamination on the surface and iii) Contamination underneath.

\section{Results and discussion}

After overnight incubation of the prepared plates in the room temperature, all the plates were analyzed for contamination. In the set A preparation, contaminations were found on the surface of 12 plates out of 25 ( $48 \%$ ). In this stage, the contaminated plates alone discarded and the remaining uncontaminated plates were used for further microbiological analysis.

In the set B, a total of 25 plates were prepared aseptically and kept for sterility checking; as a result, 20 plates (80\%) were contaminated underneath the surfaces. Thus all the contaminated and uncontaminated plates were discarded. Among the 25 plates of set C, 18 plates (72\%) were contaminated, where 8 plates (44.5\%) were contaminated on the surface, 6 plates (33.3\%) were found contaminated underneath the surface and 4 plates $(22.2 \%)$ have both surface and underneath contamination, thus all the plates were discarded (Table 1). 
Table 1 Probability of discarding contaminated plates

\begin{tabular}{|c|c|c|c|c|c|c|}
\hline Set & $\begin{array}{l}\text { Culture } \\
\text { media }\end{array}$ & $\begin{array}{l}\text { Number } \\
\text { of plates } \\
\text { prepared }\end{array}$ & $\begin{array}{l}\text { Incubati } \\
\text { on }\end{array}$ & $\begin{array}{l}\text { Number of } \\
\text { plates } \\
\text { contamina } \\
\text { ted }\end{array}$ & $\begin{array}{ll}\text { Location } & \text { of } \\
\text { contamination }\end{array}$ & Remarks \\
\hline $\mathrm{A}$ & \multirow[t]{3}{*}{$\begin{array}{l}\text { Nutrient } \\
\text { Agar }\end{array}$} & 25 & \multirow[t]{3}{*}{$\begin{array}{l}\text { Room } \\
\text { temperat } \\
\text { ure }\end{array}$} & $12(48)$ & Surface & $\begin{array}{l}\text { Only contaminated plates were } \\
\text { discarded; uncontaminated } \\
\text { plates were used }\end{array}$ \\
\hline $\mathrm{B}$ & & 25 & & $20(80)$ & Underneath surface & All plates were discarded \\
\hline $\mathrm{C}$ & & 25 & & $18(72)$ & $\begin{array}{l}8(44.5) \text { on surface } \\
6(33.3) \text { underneath } \\
4(22.2) \text { both }\end{array}$ & All plates were discarded \\
\hline
\end{tabular}

This study is an eye opener for the microbiologist for scheduling the discarding procedures by means of contamination criteria as mentioned in table. Eventhough various intrinsic and extrinsic factors responsible for microbial growth [3, $7,12-13]$, the method of sterilization and aseptic handling procedures play very important role in maintaining the culture plate sterility. Temperature is a main factor that potentially affects the microbial growth when it gets increases or decreases; thus optimizing according to the microbial character is quite essential [8, 14]. The laboratory based special criteria is also planned to evacuate the incubation period based on the growth of microbes.

As per the present study, it was suggested to prepare the culture media and utilize the plates freshly. Maintaining the plates in low temperature sometimes enhance the growth of psychrophilic organisms [9, 16-17]. Thus each and every laboratory has to analyze and validate the shelf life of the prepared agar plates, otherwise dryness leads to the plates useless. In some cases, research scholars are re-using the un-grown (inoculated) plates for other purposes. But it should not be happened because some slow growers may also found on the samples that disturb the new inoculation.

The quality of the culture media preparation without contaminations is primarily depend on the quality of the raw materials including petri dishes, chemicals used for media preparation, additive like whole egg, blood, serum etc. and, water [17]. If these parameters are analyzed properly means, then the chance of getting contamination may reduce to $50 \%$. Improper sterilization of the glassware and nutrients for preparing plates were also make the set up contaminated. If improper sterilization occurs then the underneath colonies found makes the whole set up unwanted.

Improper preparations of the plates also play a vital role in contamination like bubbles in media, cracks, unequal filling of the medium, variable amounts of medium in petridishes, color of the medium, gross deformation in the surface of the medium and storing the prepared plates in low temperature without sterility checking [18]. Along with that, handling procedures and additional supporting criteria should be properly planned like, surface cleaning of hands, usage of gloves, laminar air flow, surface cleaning of laminar chamber using chemicals and UV, and avoiding oral microbial flow (mask is the alternative).

By this way, all the prepared nutrient or enriched agar plates must be scrupulously checked for contamination batch wise. In some studies suggested that the whole batch of prepared media should be checked for contamination by placing the plates at room temperature for at least three days [5,19]; but we strongly suggested that the contamination due to poor sterility and improper handling may visualized on day one itself (18 - 24 hours). Most of the studies described that only few plates from the batch may keep for sterility checking [20]; in this juncture, we strongly oppose and suggest checking all the prepared plates for at least 24 hours and further storing in low temperature.

\section{Conclusion}

Overall from this study, the microbiological laboratories should follow certain guidelines or to pursue the subsequent stepwise analysis for sterility management including clean room design, airlock and aseptic personnel protective equipments (PPEs), clean room fittings and surfaces, regular and proper cleaning, sanitization and disinfection procedures. Further, frequent and routine environmental monitoring and appraisal, scheduled fumigation practices for maintaining air sanitation also required. The immediate disposal or cleaning of discarded materials (avoid dumping), maintaining and standardizing positive and negative control for variable and invariable sterility assessments should be adhered as per the institutional policies. 


\section{Compliance with ethical standards}

\section{Acknowledgments}

Authors are gratefully acknowledge the management of Trichy SRM Medical College Hospital and Research Centre for permitting to execute and publish this research.

\section{Disclosure of conflict of interest}

None

\section{References}

[1] Basu S, Bose C, Ojha N, Das N, Das J, Pal M and Khurana S. (2015). Evolution of bacterial and fungal growth media. Bioinformation, 11, 182-184.

[2] Rashid MI, Mujawar LH, Shahzad T, Almeelbi T, Ismail IMI and Oves M. (2016). Bacteria and fungi can contribute to nutrients bioavailability and aggregate formation in degraded soils. Microbiological Research, 183, 26-41.

[3] Elsas JDV, Semenov AV, Costa R and Trevors JT. (2011). Survival of Escherichia coli in the environment: fundamental and public health aspects. ISME Journal, 5, 173-183.

[4] Sochocka M andBoratynski J. (2011). Osmoregulation- an important parameter of bacterial growth. PostepyHig Med Dosw, 22, 714- 724.

[5] Giai C, Ortiz MR, Kappes MA, Senko J and Lannuzzi M. (2016). Efficacy of sterilization methods and their influence on the electrochemical behavior of plain carbon steel. Journal of the Electrochemical Society, 163, 633-642.

[6] Sanders ER. (2012). Aseptic laboratory techniques: plating methods. Journal of Visualized Experiments, 63, 30643081.

[7] Jett BD, Hatter KL, Huycke MM and Gilmore MS. (1997). Simplified agar plate method for quantifying viable bacteria. BioTechniques, 23,648-650.

[8] Remoundou K and Koundouri P. (2009). Environmental effect on public health; an economic perspective. International Journal of Environmental Research and Public Health, 6, 2160-2178.

[9] Adams RLP. (1980). Glassware preparation and sterilization techniques. Laboratory Techniques in Biochemistry and Molecular Biology, 8, 151-164.

[10] Jones RN, Krisher K and Bird DS. (2003). Results of the survey of the quality assurance for the commercially prepared microbiology media. Archives of Pathology and Laboratory Medicine, 127, 661-665.

[11] Krisher K. (2001). Survey results: the use of the NCCLS standard M22-A2 and Quality assurance for commercially prepared microbiologic culture media by clinical laboratories. Clinical Microbiology Newsletter, 23, 59-63.

[12] Urmila M, Poonam N, Shivani M and Kulvinder K. (2017). A growth of different types of microorganism, intrinsic and extrinsic factors of microorganism and their affects in food: a review. International Journal of Current Microbiology and Applied Sciences, 6, 290-298.

[13] Aiyegoro OA. (2014). Microbial contamination of processed meat. Encyclopedia of Meat Sciences, 19, 289-293.

[14] Rolfe MD, Rice CJ, Lucchini S, Pin C, Thompson A, Cameron ADS, Alston M, Stringer MF, Betts RP, Baranyi J, Peck MW and Hinton JCD. (2012). Lag phase is a distinct growth phase that prepares bacteria for experimental growth and involves transient metal accumulation. Journal of Bacteriology, 194, 686-701.

[15] Sandle T and Skinner K. (2012). Study of psychrophilic and psychotolerant microorganism isolated in cold rooms used for pharmaceutical processing. Journal of Applied Microbiology, 114, 1166-1174.

[16] Chattopadhyay MK. (2006). Mechanism of bacterial adaptation to low temperature. Journal of Biosciences, 31, 157-165.

[17] Cantarelli VV, Inamine E, Brodt TCZ, Secchi C, Pereira FDS, Amaro MC, BatalhaAA andLigiero SD. (2003). Quality control for Microbiological culture media. Is it enough to follow the NCCLS M22-A2 procedures? Brazilian Journal of Microbiology, 34, 8-10. 
[18] Microbiology of food, animal feed and water - Preparation, production and storage and performance testing of culture media. (2014). ISO 11133: 2014 (E), 1-95. http://www.sis.se/api/document/preview/917348.

[19] Arora DR. (2004). Quality Assurance in Microbiology. Indian Journal of Medical Microbiology, 22, 81-86.

[20] Scheme PIC. (2007). Recommendation on the Isolators used for Aseptic Processing and Sterility Testing, PI 0123,6 .

\section{How to cite this article}

Prabhusaran N, Pramila M and Anupriya A. (2018). Think before use: Sterility checking of culture plates. GSC Biological and Pharmaceutical Sciences, 5(1), 82-86. 